\title{
Krankenpflegeversicherung und Off-Label-Use: Die Ärzteschaft ist gefordert
}

\author{
Off-Label-Use birgt zahlreiche Probleme. Zum Beispiel sind Anträge auf Kostenüber- \\ nahme oft unzulänglich, über die Vergütungshöhe gibt es lange Verhandlungen \\ zwischen Versicherern und Pharmaindustrie. Im Interesse der Patienten sollte eine \\ verbindliche Regelung der Vergütungshöhe in Abhängigkeit vom Stand der Regis- \\ trierung und SL-Listung der Arzneimittel durch Swissmedic und BAG erfolgen.
}

Die obligatorische Krankenpflegeversicherung (OKP) übernimmt die Kosten für Arzneimittel, die in der Spezialitätenliste (SL) aufgelistet sind. In der SL werden nur Arzneimittel aufgelistet, die über eine Zulassung von Swissmedic verfügen. Die Zulassung durch Swissmedic umfasst die pharmazeutische Spezialität und die Fachinformation. Die registrierte pharmazeutische Spezialität muss gemäss Fachinformation angewendet werden. Jede Abweichung von der Fachinformation bei der Anwendung (u. a. andere Dosierung, andere Indikation, andere Patientengruppe) ist heilmittelrechtlich nicht registriert. Es handelt sich um «Off-Label-Use» (OLU). OLU ist erlaubt, sofern dies medizinisch bzw. wissenschaftlich begründet werden kann, der Patient ausreichend informiert wurde und der ausserordentlichen Anwendung zugestimmt hat. Die Information über OLU gehört zum Behandlungsauftrag. Die Begründung [1] muss in der Krankenakte festgehalten werden.

\section{«In der Onkologie erfolgt in bis}

\section{zu 75\% Off-Label-Use.»}

OLU ist verbreitet. So werden in der Grundversorgung [2] in über zehn Prozent pharmazeutische Spezialitäten in Abweichung von der Fachinformation eingesetzt, in mehr als drei Viertel der Fälle ohne wissenschaftliche Evidenz. In der Pädiatrie erfolgen abhängig vom Einsatzort [3] bis zu $65 \%$ der Verordnungen ausserhalb der Fachinformation, da nur wenige Arzneimittel in dieser Altersgruppe untersucht und registriert worden sind. OLU in der Pädiatrie kann meistens durch allgemein anerkannte Erfahrungen begründet werden. In der Onkologie erfolgt in bis zu 75\% OLU [4]. Es handelt sich einerseits um nach der Registrierung entdeckte und durch Erfahrung belegte Einsatzgebiete bekannter Arzneimittel, deren Patent abgelaufen ist, anderer-

\section{Assurance obligatoire des soins et utilisation hors étiquette: le corps médical est mis à contribution}

L'assurance obligatoire des soins (AOS) prend en charge les coûts des médicaments figurant dans la liste des spécialités (LS), dans laquelle ne figurent toutefois que les médicaments autorisés par Swissmedic. L'autorisation de mise sur le marché comprend la spécialité pharmaceutique et l'information professionnelle. La spécialité pharmaceutique enregistrée devant être appliquée conformément à l'information professionnelle, les écarts par rapport à cette dernière lors de l'utilisation du médicament (par ex. autre dosage, autre indication, autre groupe de patients) ne sont pas enregistrés dans la législation sur les produits thérapeutiques. On parle dès lors $d^{\prime}$ «Off-Label-Use». Cette utilisation «hors étiquette» est autorisée pour autant qu'elle soit médicalement et scientifiquement motivée, que le patient ait été suffisamment informé et qu'il ait donné son accord à cette utilisation exceptionnelle. L'information sur l'utilisation hors étiquette fait partie du mandat thérapeutique et sa justification doit être inscrite dans le dossier médical.

seits um den Einsatz neuerer Arzneimittel in Zweitund Drittlinientherapien oder in neuen Indikationen, die auf neuen, oft vorläufigen Resultaten, die an internationalen Kongressen mündlich oder als Poster präsentiert wurden, beruhen. In der Onkologie und bei seltenen Krankheiten kann bei fehlenden therapeutischen Alternativen in Einzelfällen der Einsatz eines nichtregistrierten Arzneimittels medi- 
zinisch begründet angebracht sein. Es handelt sich um «Unlicensed-Use».

OLU und Unlicensed-Use werden im Prinzip von der OKP nicht übernommen. Auch darüber muss der Patient orientiert werden. OLU von auf der SL gelisteter Arzneimittel melden Ärzte selten den Krankenversicherern. Es darf davon ausgegangen werden, dass die Versicherer OLU in der Pädiatrie im Allgemeinen stillschweigend akzeptieren. Bei neueren, meistens hochpreisigen Arzneimitteln erfolgen vermehrt Kontrollen, die zu Nachfragen bei den behandelnden Ärzten führen. Falls die Versicherer die Kosten solcher Arzneimittel nicht übernehmen, müssen sicherung (KVV). Diese Artikel umschreiben die Voraussetzungen zur Übernahme der Kosten im Einzelfall einerseits auf der SL gelisteter Arzneimittel für OLU und für Einsatz ausserhalb der SL-Limitation und andererseits nicht auf der SL gelisteter Arzneimittel durch die OKP. Die Übernahme der Kosten dieser Arzneimittel muss gemäss den Artikeln bejaht werden, wenn deren Einsatz eine unerlässliche Voraussetzung für die Durchführung einer anderen übernommenen Leistung ist, oder wenn vom Einsatz dieses Arzneimittels ein grosser therapeutischer Nutzen gegen eine Krankheit, die für die Patientin oder den Patienten tödlich verlaufen oder schwere

\section{Ärzte reichen oft medizinisch ungenügende bzw. wissenschaftlich nicht evidente Unterlagen für die Beurteilung der Kostenübernahme ein.}

diese durch die behandelnden Ärztinnen und Ärzte übernommen werden. Es muss daher dringend geraten werden, beim Versicherer zuhanden des Vertrauensarztes vorgängig ein medizinisch-wissenschaftlich begründetes Gesuch um Übernahme der Kosten dieses Arzneimittels für die individuelle Patientin einzureichen. Diese empfohlene Vorgehensweise betrifft vor allem den Einsatz auf der SL gelisteter Arzneimittel ausserhalb der Fachinformation, ausserhalb der in der SL genannten Limitation und aller nicht auf der SL gelisteten Arzneimittel im Einzelfall.

Um die Kostenübernahme im Einzelfall durch die OKP zu vereinheitlichen bzw. zu regeln, erliess der Bundesrat die Art. 71 a und $71 \mathrm{~b}$ (in Kraft seit 1. März 2011) der Verordnung über die Krankenver-

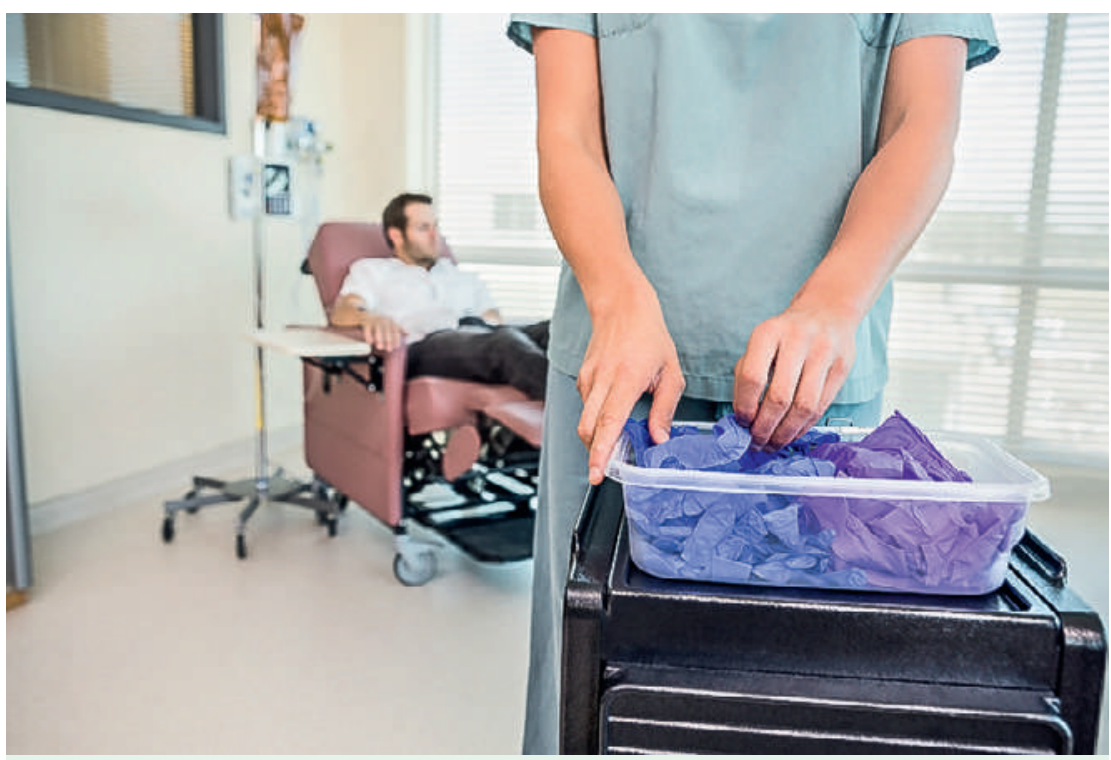

Besonders häufig in der Onkologie: Off-Label-Use. Er ist erlaubt, sofern er medizinisch bzw. wissenschaftlich begründet werden kann. und chronische gesundheitliche Beeinträchtigungen nach sich ziehen kann, erwartet wird und wegen fehlender therapeutischer Alternativen keine andere wirksame und zugelassene Behandlungsmethode verfügbar ist.

Die Umsetzung der KVV-Artikel durch die Krankenversicherer führte in Einzelfällen zu Ungleichbehandlung, die Nationalrat Steiert zur Einreichung einer Motion veranlasste, die vom Nationalrat am 21. März 2013 und vom Ständerat am 4. März 2014 überwiesen wurde. Die Motion verlangt, dass der Bundesrat beauftragt werde, die Verordnung über die Krankenversicherung dahingehend zu ändern, dass die Vergütung von Arzneimitteln, die nicht von Swissmedic zugelassen oder nicht vom BAG in die SL aufgenommen worden sind, wohnort- und versicherungsunabhängig geregelt wird. Die Motion veranlasste das BAG zur Ausschreibung einer Studie zur Umsetzung der KVV.

Die Studie [5] wurde mittels einer schriftlichen Umfrage bei den Krankenversicherern und vertrauensärztlichen Diensten durchgeführt. Die Studie weist gewichtige Mängel auf. So musste beispielsweise leider mehrheitlich auf retrospektive und unvollständige Daten zurückgegriffen werden, da nur vereinzelte Krankenversicherer und vertrauensärztliche Dienste die Daten prospektiv und vollständig erfassen. Zudem lieferten nur 28 von 61 Krankenversicherern Daten. Weiter wurden einzig 15 vertrauensärztliche Dienste befragt. Die Arzneimittel - sowohl pharmazeutische Spezialität als auch Wirksubstanz - wurden nicht erfasst. Deshalb kann keine fundierte Aussage gemacht werden über die Krankheitsgebiete (u.a. Rheumatologie, seltene Krankheiten, bösartige Erkrankungen), in denen ein ausserordentlicher Einsatz im Einzelfall erfolgte. So streuten die von den Versicherern gelieferten Zahlen über die Verteilung der Gesuche auf Therapiegebiete stark. 
Für die Onkologie zum Beispiel erstreckten sich diese von 25 bis 90\% der gemeldeten Gesuche. Auch kann die Qualität des ausserordentlichen Einsatzes der Arzneimittel nicht beurteilt werden. Schliesslich bleibt zu erwähnen, dass pro Jahr schätzungsweise 6000-8000 Gesuche um Kostenübernahme der Arzneimittel im Einzelfall bei den Krankenversicherern eingehen, weniger als ein Gesuch pro 1000 Versicherte.

Die Studie zeigt trotz der genannten Mängel drei wichtige Punkte auf:

1. Die behandelnden Ärztinnen und Ärzte reichen oft medizinisch ungenügende bzw. wissenschaftlich nicht evidente Unterlagen für die Beurteilung der Kostenübernahme im Einzelfall ein. Der kritische Leser fragt sich, wie gut der ausserordentliche Einsatz der Arzneimittel medizinisch belegt werden kann und wie gut die Ärztinnen und Ärzte die Patientinnen und Patienten darüber informierten. Die Ärzteschaft ist hier gefordert: Die Aufklärung der Patientinnen und schliessende Beurteilung des spezifischen Einzelfalls. Die Vertrauensärzte sollten die Gesuche und die medizinischen Beurteilungen prospektiv und vollständig erfassen. Das Schaffen einer internetbasierten Plattform ermöglicht im Interesse der Qualität der Patientenversorgung, Wissen über den ausserordentlichen Arzneimitteleinsatz zu gewinnen.

3. Die Festsetzung der Kosten für den ausserordentlichen Einsatz von Arzneimitteln führte oft zu langwierigen Verhandlungen zwischen Krankenversicherern und der Pharmaindustrie. Im Interesse der Patientinnen und Patienten sollte eine verbindliche Regelung der Vergütungshöhe in der KVV in Abhängigkeit vom Stand der Registrierung und SL-Listung der Arzneimittel durch Swissmedic und BAG erfolgen. Die Vergütungshöhe für neue nichtangemeldete Indikationen in der SL gelisteter Arzneimittel sollte deutlich unterhalb des vom BAG verfügten Fabrikabgabepreises liegen. Die Pharmaindustrie ist hier ge-

\section{Es braucht eine verbindliche Regelung der Vergütungshöhe in der Verordnung über die Krankenversicherung, abhängig vom Stand der Registrierung und SL-Listung der Arzneimittel durch Swissmedic und BAG.}

Patienten über jeden ausserordentlichen Einsatz von Arzneimitteln (u.a. ausserhalb der SL oder ausserhalb der Fachinformation oder nichtregistrierte Arzneimittel) gehört zu ihrem Behandlungsauftrag. Es ist den gesuchstellenden Ärztinnen und Ärzten dringend zu empfehlen, vorgängig der Information der Patienten den hohen Nutzen des möglichen ausserordentlichen Arzneimitteleinsatzes selbst mit dem Modell der Gesellschaft der Vertrauensärzte zu beurteilen. Die Patientinnen und Patienten müssen verständlich informiert werden und dem Einsatz zustimmen. Erst dann kann das medizinisch und wissenschaftlich gut begründete Gesuch um Kostenübernahme zusammen mit allfälligen wissenschaftlichen Unterlagen zuhanden des Vertrauensarztes an den Krankenversicherer gesandt werden.

2. Die Vertrauensärztinnen und Vertrauensärzte wenden am häufigsten das 9-Felder-Modell, gefolgt vom MediScore-Modell, zur Beurteilung des hohen Nutzens bzw. der Zweckmässigkeit der ausserordentlich eingesetzten Arzneimittel an. Die Gesellschaft der Vertrauensärzte ist hier gefordert: Es sollte wenn immer möglich ein einziges Modell zur Nutzenbeurteilung [6] angewendet werden. Darauf basierend erfolgt die ab- fordert: Die Arzneimittel-Fachinformationen sollten bei Indikationserweiterungen durch die Pharmafirmen möglichst rasch angepasst und dem BAG zur Aufnahme in die SL gemeldet werden. Interne Daten über OLU von Arzneimitteln sollten Fachpersonen über eine internetbasierte Plattform zugänglich gemacht werden.

Die Erfüllung der genannten Forderungen wird den Weg zum gleichen Zugang für alle Versicherten zu Arzneimitteln ermöglichen.

\section{Literatur}

1 Giger M, Saxer U, Wildi A, Fritz MB. Arzneimittelrecht. Zürich: Schulthess; 2013.

2 Eguale T. et al. Drug, patient, and physician characteristics associated with off-label prescribing in primary care. Arch Intern Med. 2012;172:781-8.

3 Kimland E. Odlind V. Off-label drug use in pediatric patients. Clin Pharmacol Ther. 2012;91:796-801.

4 Soares M. Off-label indications for oncology drug use and drug compendia: history and current status. J Oncol Practice. 2005;1:102-5.

5 Rüefli C, Bolliger C. Off-Label-Use in der obligatorischen Krankenpflegeversicherung. Evaluation der Umsetzung von Artikel 71a und 71b KVV. Bern: Bundesamt für Gesundheit. 2013.

6 www.vertrauensaerzte.ch/expertcom/71kvv/ 